\title{
GÊNERO E PSICOLOGIA SOCIAL NO BRASIL: ENTRE SILÊNCIO E DIÁLOGO
}

\author{
Jaqueline Gomes de Jesus \\ Centro Universitário Planalto do Distrito Federal - UNIPLAN - Brasil
}

Ana Lúcia Galinkin

Universidade de Brasília - UnB - Brasil

\begin{abstract}
Resumo
Com o impacto das teorias feministas no ambiente acadêmico brasileiro, e sua crítica a modelos tradicionais de investigação, o conceito de gênero tem sido cada vez mais abordado dentro dos diferentes ramos da ciência psicológica, mesmo que ainda seja tratado, de forma geral, apenas como uma variável, geralmente confundida com o sexo biológico, e não como um eixo estruturante de estudos sobre as relações sociais. O presente trabalho apresenta um estudo exploratório, com base em revisão bibliográfica de artigos científicos, sobre os usos do conceito de gênero na Psicologia Social brasileira. Foram identificados apenas 32 artigos na área de Psicologia Social em um universo de 161.363 artigos sobre gênero. Os resultados indicam que há poucos estudos de gênero focados nas questões psicossociais $(0,01 \%$ da produção científica). Conclui-se que há a necessidade de estimular estudos de gênero em Psicologia Social, a fim de explicitar os discursos psicossociais acerca dos sujeitos e das relações grupais, com enfoque de gênero.
\end{abstract}

Palavras-chave: Estudos de gênero. Psicologia social. Produção científica.

\section{Introdução}

O conceito contemporâneo de "gênero", como consequência de sua funcionalidade (SCOTT, 1995), abarca diferentes campos do saber. Entretanto, nem todas as áreas do conhecimento científico têm apreciado, analisado e aplicado o conceito em sua complexidade, destacando-se nos estudos de gênero as ciências sociais, em termos de número e qualidade dos trabalhos. 
A Psicologia Social têm produzido saberes sobre gênero aquém de suas possibilidades, apesar de se constituir enquanto um campo de referência com olhar privilegiado para as interações e expectativas de interações entre as pessoas (RODRIGUES, ASSMAR \& JABLONSKI, 2003), e da sua marca distintiva frente às demais ciências sociais, por ser constituída a partir de uma unidade de análise com enfoque nos indivíduos, característica essa que permite encontrar respostas para perguntas diferentes das comumente feitas por outras ciências ou mesmo pela própria Psicologia (LANE \& CODO, 1984 $4^{1}$ ).

Os problemas decorrentes dessa pouca participação não são apenas teóricos e empíricos (o que por si só bastaria para suscitar preocupação no que tange à busca por conhecimento), eles repercutem no fato de que, havendo um número razoável de estudos antropológicos e sociológicos, pouco se sabe "psicossocialmente" sobre gênero, e isso pode ser desastroso em termos práticos.

Considerando que raramente nos indagamos sobre a natureza das questões de gênero e sua interferência em nosso cotidiano, perguntas como: "o que é gênero?”, "o que/quem é um homem?”, “o que/quem é uma mulher?” ou “ela/ele/isso é um/a mulher/homem?”, podem ser "facilmente" respondidas no âmbito da cultura de massas - os meios de comunicação contemporâneos são um exemplo dos discursos categóricos das culturas para defender o que seria um homem e uma mulher "de verdade", senão modelos arquetípicos de ideais fisionômicos e atitudinais (JESUS, 2012a) —, entretanto, não são respondidas tão habilmente se levados em conta os indivíduos e seus diferentes comportamentos frente à cultura.

Afirmar isso corresponde a constatar, por um lado, que prevalecem noções superficiais acerca dos processos identitários estruturantes de identidades sexuais/de gênero, em geral confundidas com perspectivas socioculturais estereotipadas, tese predominante na Psicologia, que, no entanto, vem sendo contestada por algumas psicólogas desde o século XIX (GALINKIN, SANTOS \& ZAULI-FELLOWS, 2010).

No entanto, explicar toda expressão de gênero a partir unicamente da cultura é simplificar o conceito: se tudo é cultura, nada é cultura. A Psicologia detém instrumental para entendimento de determinados fenômenos culturais, como gênero, que falta a outras ciências sociais, particularmente "o lado pessoal das experiências vividas dentro da cultura, quer seja 'na cultura' ou 'tendo cultura', conforme os próprios sentimentos e pensamentos" (VALSINER, 2012, p. 27).

\footnotetext{
${ }^{1}$ Lane, S. T. M., \& Codo, W. (1984). Psicologia social: o homem em movimento. São Paulo: Brasiliense.
} 
Paradoxalmente, apesar de ser identificada como uma profissão feminina, consideração decorrente da predominância de psicólogas (CASTRO \& YAMAMOTO, 1998), a Psicologia, como ciência, tem envidado menos esforços nos estudos de gênero do que as Ciências Sociais, e sua abordagem das questões de gênero, quando acontece, geralmente é orientada a partir dos estereótipos do senso comum que relacionam diferenças biológicas com diferenças psicossociais, classificados por Bem (1994) como: 1) polarização de gênero (mulheres e homens teriam papéis mutuamente excludentes); 2) androcentrismo (a experiência masculina seria o padrão neutro ou norma); e 3 ) essencialismo biologicista (os comportamentos seriam consequências naturais e inevitáveis de naturezas biológicas intrínsecas).

Psicólogos que se orientam por uma leitura feminista da realidade têm criticado o reducionismo biológico presente na Psicologia, buscando ressaltar as semelhanças entre homens e mulheres e identificar os determinantes ambientais das suas diferenças (CRAWFORD \& UNGER, 2004).

Talvez pela proximidade com as Ciências Sociais, a Psicologia Social seja um ramo da Psicologia com maiores potencialidades, relativamente ao tema "gênero", de superar a visão mainstream supracitada, adotando uma perspectiva sobre gênero que reconheça o seu contexto sócio-histórico e cultural.

Entretanto, apesar de seu potencial, não existem, no Brasil, manuais de Psicologia Social que tratem especificamente do assunto "gênero", reservando-lhe, por exemplo, um capítulo próprio.

\section{Estudos de gênero e psicologia social}

Consultados doze livros em língua portuguesa, ou traduzidos para o português, que objetivam introduzir a disciplina Psicologia Social para estudantes, publicados no Brasil ao longo de um período de 37 anos, de 1974 a 2011 (KATZ \& KAHN, 1974²; LAMBERT \& LAMBERT, 1975³ ; MAISONNEUVE, 1977²; LANE \& CODO, 1984; ARONSON, WILSON \& AKERT, 2002; FURTADO, BOCK \& TEIXEIRA, 2002; JACQUES, STREY, BERNARDES, GUARESCHI, CARLOS \& FONSECA, 2002; RODRIGUES, ASSMAR \&

\footnotetext{
${ }^{2}$ Katz, D., \& Kahn, R. (1974). Psicologia social das organizações. São Paulo: Atlas.

${ }^{3}$ Lambert, W., \& Lambert, W. (1975). Psicologia social. Rio de Janeiro: Zahar.

${ }^{4}$ Maisonneuve, J. (1977). Introdução à psicossociologia. São Paulo: Editora Nacional / Editora da Universidade de São Paulo.
} 
JABLONSKI, 2003; LANE, 20065' ÁLVARO \& GARRIDO, 2007; TORRES \& NEIVA, 2011), constata-se que apenas um deles (JACQUES, STREY, BERNARDES, GUARESCHI, CARLOS \& FONSECA, 2002) apresenta um capítulo específico para o estudo do gênero no campo da Psicologia.

Tamanha cegueira teórica às questões de gênero é contrária ao exemplo dado pela quarta edição do clássico Handbook of Social Psychology, de Gilbert, Fiske e Lindzey, de 1998, que tratou gênero como "um construto psicossocial significativo (...) que abrange desde crenças e ações individuais até o impacto de sistemas sociais" (DEAUX \& LAFRANCE, 1998, p. 788).

Esse fato aponta para uma realidade: a formação dos psicólogos sociais brasileiros em gênero, atualmente, demanda leituras em outras línguas e, principalmente, em outras áreas do conhecimento, o que, mesmo sendo desejável do ponto de vista do pensamento transdisciplinar, característico dos estudos de gênero, aponta para a falta de interesse em produção do conhecimento sobre gênero a partir do olhar científico psicossocial.

Procedendo a uma leitura da formação histórica da Psicologia Social moderna se encontram sustentáculos para a predileção de seus profissionais a certos questionamentos em detrimento de outros, como os relacionados a gênero.

Ciência tida como de longa existência como pensamento, porém com breve história como disciplina independente (MARKOVÁ \& MOSCOVICI, 2006), desenvolveu-se nos Estados Unidos e na Europa, ao longo das duas décadas seguintes à Segunda Guerra Mundial, em um fluxo de conhecimentos mediado por circunstâncias políticas e históricas particulares, que determinou os temas científicos essenciais para a Psicologia Social em suas vertentes conhecidas: a Social psicológica, de cunho metodológico experimental e marcadamente influenciada por autores norte-americanos; e a Social sociológica, tipificada pela metodologia sócio-histórica, de forte influência europeia (BERNARDES, 2002).

A Psicologia Social psicológica floresceu no período pós-guerra supracitado, com repercussões acadêmicas no Brasil, variando da reprodução de posturas teórico-metodológicas à crítica dessa práxis, principalmente na década de 1970, momento marcado por graves circunstâncias políticas na América Latina, porém pouco tratado por tal vertente, daí a valorização subsequente do modelo psicológico europeu (social sociológico), considerado mais adequado para o estudo dos problemas de interesse de então.

\footnotetext{
${ }^{5}$ Lane, S. T. M. (2006). O que é psicologia social. São Paulo: Brasiliense.
} 
Como aponta Perucchi (2009), a aplicação do conceito de gênero na Psicologia Social, não apenas como uma variável em um modelo experimental, deu-se em meio a descrédito por parte da comunidade acadêmica.

Para além do fortalecimento de instituições ligadas à Psicologia Social sociológica, tal conjuntura intelectual retroalimentou inclusive o foco de seu contradiscurso, o que se pode verificar nos anos1980 com estudos sociais psicológicos que sutilmente distinguiam psicologias sociais e psicologias sociais "científicas", e que buscavam demonstrar que conhecimentos experimentais podem ser investidos no contexto social, a exemplo de um artigo de Rodrigues (1986) que defende a contribuição da Psicologia Social para a resolução de conflitos internacionais.

Esse período coincide com a terceira fase dos estudos de gênero no Brasil, datada por Costa, Barroso e Sarti (1985), na qual, após 1985, começavam a ser elaboradas teorias que associavam gênero e relações de dominação/subordinação. No contexto da Psicologia, predominantemente social psicológico, o conceito de gênero era ainda tratado de forma periférica, restrito à variável "sexo", de base biológica, fator determinante de diferenças entre os gêneros encontradas em condições experimentais (STREY, 2002).

Nos anos 1990, tal silêncio teórico sobre gênero foi afetado pela ascensão da vertente social sociológica e o diálogo crescente da sociedade civil organizada com as Ciências Sociais. O movimento feminista teve uma especial interlocução com a Antropologia, a Sociologia e a História, mas, quanto à Psicologia, esse diálogo se restringiu a alguns olhares quanto a simplistas diferenças sexuais "entranhadas na linguagem, métodos e orientações cognitivas da Psicologia" (MORAWSKI, 1994, p. 21).

Pode-se identificar nesse período uma ampliação da agenda de estudos de gênero na Psicologia Social, com a inclusão de estudos sobre masculinidade e, devido à discussão com o movimento de Lésbicas, Gays, Bissexuais, Travestis e Transexuais - LGBT, sobre orientação sexual e questões de gênero aplicadas às diferentes realidades dessas populações (GALINKIN \& ZAULI-FELLOWS, 2010).

Desde a primeira década no Século XXI, apesar de avanços, ainda é frágil a presença das questões de gênero na Psicologia Social brasileira, representada por livros como o de Fonseca (2000), que aborda gênero na perspectiva da subjetividade e do trabalho, o de Galinkin e Santos (2010), que apresenta interfaces da Psicologia Social com gênero, o comemorativo dos 30 anos da Associação Brasileira de Psicologia Social - ABRAPSO (MEDRADO \& GALINDO, 2011), o qual abriga dois capítulos sobre gênero e feminismos de grande impacto (TONELI \& AMARAL, 2011; BENTO, 2011), mesmo não sendo escritos por 
psicólogas sociais (duas autoras são psicólogas escolares e do desenvolvimento e a terceira é socióloga), o que, concomitantemente, sinaliza para a falta de psicólogos sociais estudando gênero, ou a sua invisibilidade.

O quadro se agrava ao ser considerado que o mundo político e institucional sofre transformações profundas em sua tessitura, decorrentes de conquistas estruturais de grupos sociais historicamente discriminados, como cotas nas organizações para pessoas com deficiências e para mulheres nos partidos políticos, ações afirmativas para negros e indígenas no ensino superior, garantia da união civil e do casamento entre pessoas do mesmo sexo, além das lutas pela descriminalização do aborto e pela adequação do registro civil de pessoas transexuais (JESUS, 2012b).

Um elemento essencial deste início de século, particularmente de sua segunda década, é a desconstrução do modelo idealizado de mulher, desvinculado das variáveis psicossociais de construção e manutenção dos gêneros, que até então pautara os movimentos feministas e os estudos de gênero, o que tem possibilitado reconhecer, discutir e estudar outras identidades de gênero e feminismos (BENTO, 2006; JESUS \& ALVES, 2010).

Galinkin e Ismael (2010) consideram que uma das características da novíssima Psicologia Social do Gênero é a desnaturalização das diferenças entre os gêneros ao localizálas, quando são reconhecidas, no contexto histórico da cultura e do grupo humano onde

ocorrem. É a partir desse horizonte teórico que temas inicialmente abordados nos anos 1990, como masculinidade, homossexualidade e transexualidade, são aprofundados, complementando o conteúdo dos estudos sobre gênero.

Em suma, é importante conhecer a inteligentzia psicossocial em gênero existente. Assim, esta pesquisa investiga o panorama da produção científica contemporânea da Psicologia Social brasileira com foco em gênero.

\section{Método}

Foi realizada uma pesquisa quantitativa, com base em revisão bibliográfica de artigos, de autoria de psicólogos sociais, publicados em revistas nacionais no período de 24 anos entre 1988 e 2012, compreendendo o período da terceira onda dos estudos de gênero no Brasil (COSTA, BARROSO \& SARTI, 1985) e o início do século XXI.

O meio utilizado para averiguação desses dados foi a internet, cujos arquivos virtuais abrigam praticamente a totalidade do conhecimento científico e significativo publicado no país, o que, no que tange a metodologia de pesquisa na internet, aumenta a heterogeneidade 
da amostra e sua representatividade em relação ao universo da pesquisa, o que, por conseguinte, melhora a pertinência e o grau de generalização dos resultados (FRAGOSO, RECUERO \& AMARAL, 2012).

Foram empreendidas buscas nos mecanismos virtuais especializados Google (www.google.com), Scielo (www.scielo.br), Biblioteca virtual em saúde (www.bvspsi.org.br) e no portal de dissertações e teses da CAPES (www.periodicos.capes.gov.br), com base nos termos principais "Psicologia Social" e "Gênero" e nos termos secundários "Mulher", "Homem", "Homossexualidade" e "Transexualidade".

\section{Resultados}

Como resultado global, por meio do Google, do Scielo, da Biblioteca Virtual em Saúde e do portal da CAPES foram encontrados 161.363 artigos com referências aos termos buscados, destes, após análise detalhada, identificaram-se 43 com autoria de psicólogos sociais que se referiam a estudos de gênero, o correspondente a menos de $0,01 \%$ das produções intelectuais sobre gênero encontradas.

Analisando os dados provenientes dos artigos, conclui-se que, dos 108 autores, a maioria é composta por mulheres $(\mathrm{n}=91, \mathrm{p}=84 \%)$, apesar da participação significativa de homens $(\mathrm{n}=17, \mathrm{p}=16 \%)$, conforme a figura 1 .

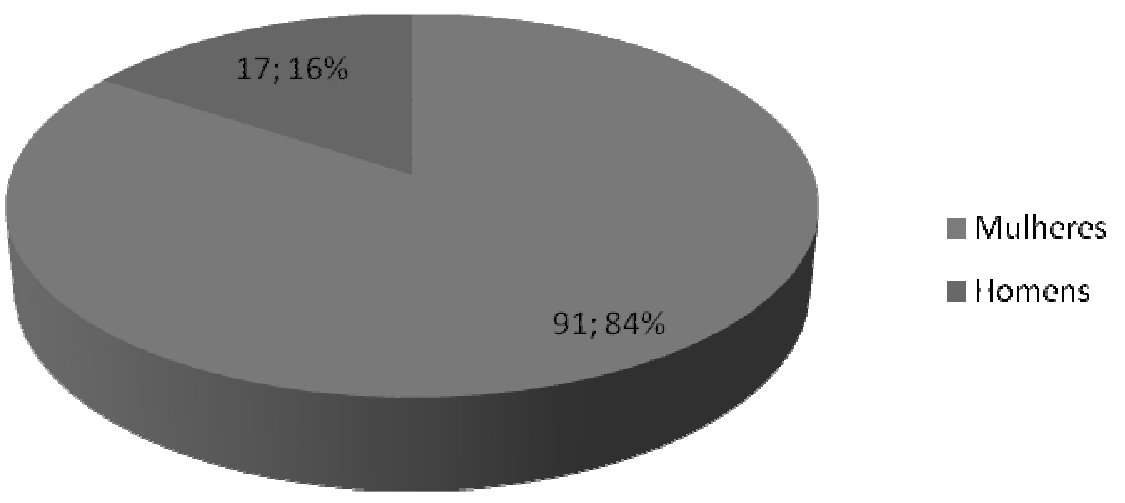

Figura 1 - Distribuição de gênero dos autores. 
Os autores se distribuem por 37 Instituições de Ensino Superior - IES, sendo 30 nacionais $(81 \%)$ e sete estrangeiras (19\%). Das IES nacionais, 20 são públicas $(67 \%)$ e 10 privadas $(33 \%)$, conforme a Figura 2.

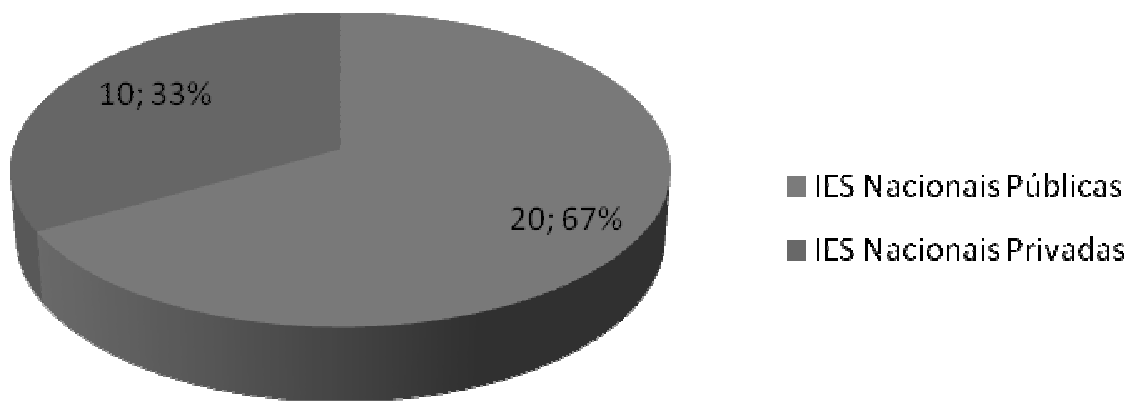

Figura 2 - Distribuição das IES nacionais representadas.

Destacam-se como instituições proeminentes na formação de pesquisadores em gênero da Psicologia Social no Brasil, com artigos publicados na temática de gênero: a Universidade de Brasília - UnB (10 autores), a Universidade Federal da Paraíba - UFPB (6 autores), a Universidade Federal do Rio Grande do Sul - UFRGS (6 autores), a Universidade Federal de Santa Catarina - UFSC (5 autores), a Pontifícia Universidade Católica do Rio Grande do Sul - PUC-RS (4 autores) e a Universidade Federal do Pará - UFPA (3 autores).

Entre as instituições estrangeiras, se destaca o Instituto Superior de Ciências do Trabalho e da Empresa - ISCTE de Portugal (3 autores). Constatou-se a pulverização de uma ou duas citações para muitas instituições: 68\% dos 108 autores são oriundos de instituições referenciadas duas vezes ou uma única vez, conforme a Figura 3. 


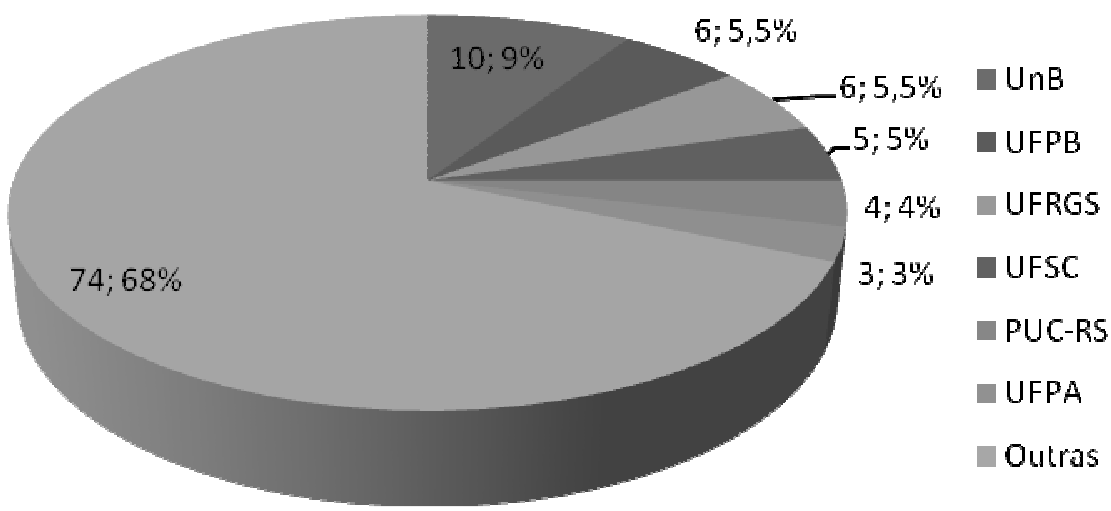

Figura 3 - Distribuição dos autores por instituições.

Procedendo-se uma distribuição dos 43 artigos de acordo com o ano de sua publicação, nota-se que 7\% são dos fins dos anos 1980 ao início do século XXI, 74,4\% são da primeira década do século XXI e 18,6\% do início da segunda década até 2012, conforme a Figura 4.

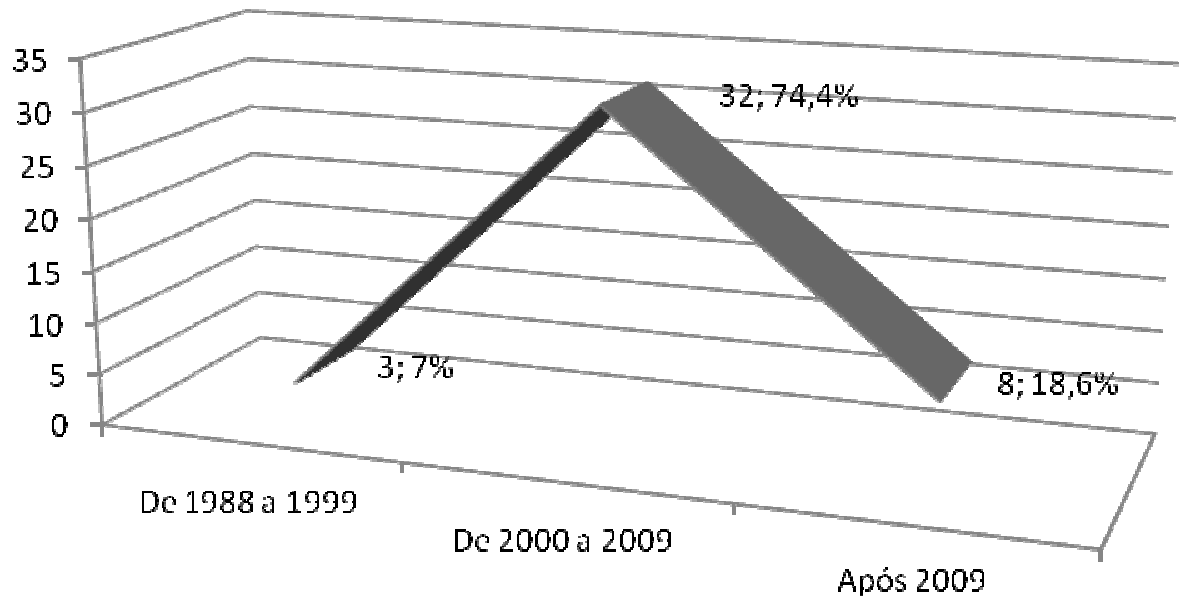

Figura 4 - Distribuição dos artigos por período.

É notável o salto de publicações sobre gênero, na Psicologia Social, da última década do século XX para a primeira década do século XXI. 


\section{Considerações finais: estudos psicossociais de gênero em revista}

Constatou-se a produção exígua de artigos acerca de gênero no campo da Psicologia Social. Tal resultado é alarmante, tendo em vista a importância de artigos científicos para a promoção de novos estudos e para o aprofundamento da área em questão nos seus tópicos de interesse particular.

Dissertações de mestrado e teses de doutorado poderiam apresentar um panorama diferente da questão, em uma pesquisa que porventura venha a ser feita, porém, devido ao fato de não serem as principais leituras utilizadas no meio universitário brasileiro, não se poderia afirmar, ante a um eventual número elevado de teses e dissertações sobre gênero na área de Psicologia Social, que estudantes estariam sendo formados e novos estudos desenvolvidos com base nessa forma de divulgação científica.

Quando da análise dos resultados quanto à distribuição dos artigos ao longo do tempo, é importante considerar que o presente estudo buscou artigos até o ano de 2012, de modo que se fazem necessárias pesquisas futuras para uma análise mais aprofundada da tendência de publicação na segunda década do século XXI.

Aventa-se que o presente artigo, em função de ter utilizado a internet como meio para encontrar os textos, possa ter tido acesso limitado a outros materiais que não os aqui citados. Em contrapartida, como recurso mais utilizado pela população em geral e pela comunidade acadêmica para pesquisas, o ambiente virtual é espaço de destaque para o que é publicado e conhecido sobre qualquer assunto, de modo que, faltando referências neste trabalho, essas ausências novamente indiciam a fragilidade com que se investe em questões de gênero no contexto da ciência psicológica social.

Pretende-se que esta pesquisa, por meio da conjuntura que se buscou retratar, contribua para um exame mais elaborado da realidade mediada pelo gênero, um olhar sem o qual as relações pautadas pelas construções culturais sobre as diferenças e semelhanças entre homens e mulheres ainda permanecem como mistérios, envoltos na névoa da incompreensão científica sobre o seu funcionamento. 


\title{
GENDER AND SOCIAL PSYCHOLOGY IN BRAZIL: BETWEEN SILENCE AND DIALOGUE
}

\begin{abstract}
With the impact of feminist theories in Brazilian academy, and its critique to traditional models of research, the concept of gender has been increasingly discussed within the different branches of psychological science, even if it is generally treated just as a variable, usually confused with the biological sex, and not as a structural axis for studies on social relations. This paper presents an exploratory study, based on a review of scientific literature on the uses of the concept of gender in Brazilian Social Psychology. They were identified only 32 articles in the field of Social Psychology, in a universe of 161.363 articles on gender. The results indicate that there is few gender studies focused on psychosocial issues $(0.01 \%$ of the scientific production). It is concluded that there is a need to encourage gender studies in Social Psychology in order to explain the psychosocial speeches about the subjects and the group relationships, with gender focus.
\end{abstract}

Keywords: Gender studies. Social psychology. Scientific production.

\section{GÉNERO Y PSICOLOGÍA SOCIAL EN BRASIL: ENTRE EL SILENCIO Y EL DIÁLOGO}

\begin{abstract}
Resumen
Con el impacto de las teorías feministas en la academia brasileña, y su crítica a los modelos tradicionales de investigación, el concepto de género se ha discutido cada vez más dentro de las diferentes ramas de la ciencia psicológica, a pesar de seguir siendo tratado, en general, al igual que una variable, a menudo confundido con el sexo biológico, y no como un estudio estructurado de las relaciones sociales. Este artículo presenta un estudio exploratorio, basado en una revisión de la literatura científica sobre los usos del concepto de género en Psicología Social en Brasil. Fueron identificados 32 artículos en el campo de la Psicología Social en un universo de 161.363 artículos en género. Los resultados indican que hay pocos estudios de género centrados en los problemas psicosociales $(0,01 \%$ de la producción científica). Se concluye que existe la necesidad de estimular los estudios de género en la Psicología Social con el fin de explicitar los discursos psicológicos sobre los sujetos y las relaciones de grupo, con una perspectiva de género.
\end{abstract}

Palabras clave: Estudios de género. Psicología social. Producción científica.

\section{Referências}

ÁlVARO, J. L., \& GARRIDO, A. Psicologia social: perspectivas psicológicas e sociológicas. São Paulo: McGraw-Hill, 2007. 
ARONSON, E., WILSON, T., \& AKERT, R. Psicologia social. Rio de Janeiro: LTC Editora, 2002 .

BEM, S. L. The lenses of gender. New Haven: Yale University,1993.

BENTO, B. A reinvenção do corpo. Rio de Janeiro: Garamond Universitária, 2006.

BENTO, B. Politizar o abjeto: dos femininos aos feminismos. In B. MEDRADO \& W. GALINDO (Orgs.), Psicologia social e seus movimentos: 30 anos de ABRAPSO. Recife: Editora Universitária da UFPE, 2011.

BERNARDES, J. S. História. In M. G. C. JACQUES, M. N. STREY, N. M. G. BERNARDES, P. A. GUARESCHI, S. A. CARLOS \& T. M. G. FONSECA (Orgs.), Psicologia social contemporânea. Petrópolis: Vozes, 2002.

CASTRO, A. E. F., \& YAMAMOTO, O. H. A Psicologia como profissão feminina: apontamentos para estudo. Estudos de Psicologia, n. 3, v.1, 147-158, 1988.

COSTA, A., BARROSO, C., \& SARTI, C. Pesquisa sobre mulher no Brasil: do limbo ao gueto? Cadernos de Pesquisa, 54, 5-15, 1985.

CRAWFORD, M., \& UNGER, R. K. Women and gender - a feminist psychology. New York: McGraw-Hill, 2004.

DEAUX, K., \& LAFRANCE, M. Gender. In D. Gilbert, S. Fiske \& G. Lindzey (Orgs.), The handbook of social psychology. New York: McGraw-Hill, 1998.

FONSECA, T. M. G. Gênero, subjetividade e trabalho. Petrópolis: Vozes, 2000.

FRAGOSO, S., RECUERO, R., \& AMARAL, A. Métodos de pesquisa para internet. Porto Alegre: Sulina, 2012.

FURTADO, O., BOCK, A. M., \& TEIXEIRA, M. L. Psicologias: uma introdução ao estudo da psicologia. São Paulo: Saraiva, 2002.

GALINKIN, A. L., \& ISMAEL, E. Gênero. In L. CAMINO, M. E. PEREIRA, M. E. O. LIMA, \& A. R. R. TORRES (Orgs.), Psicologia social: temas e teorias. Brasília: Technopolitik, 2011.

GALINKIN, A. L., \& SANTOS, C. Gênero e psicologia social: interfaces. Brasília: Technopolitik, 2010.

GALINKIN, A. L., \& SANTOS, C., \& ZAULI-FELLOWS, A. Estudos de gênero na psicologia social. In Galinkin, A. L., \& Santos, C. (Orgs.), Gênero e psicologia social: interfaces. Brasília: Technopolitik, 2010.

JESUS, J. G. A negação do corpo feminino. Mídia \& Política, n. 2, 1-3, 2012a. Recuperado em 2 agosto, de 2013, disponível em: http://www.midiaepolitica.unb.br/index.php?option=com_content $\&$ view=article \&id=85:anegacao-do-corpo-feminino\&catid=14:edicao-022012. 
JESUS, J. G. Psicologia social e movimentos sociais: uma revisão contextualizada. Psicologia e Saber Social, 1(2), 163-186, 2012b. Recuperado em 2 ago, 2013, disponível em http://www.e-publicacoes.uerj.br/index.php/psi-sabersocial/article/view/4897.

JESUS, J. G., \& ALVES, H. Feminismo transgênero e movimentos de mulheres transexuais. Cronos, 11(2), 8-19, 2010. Recuperado em 2 ago 2013, disponível em http://www.periodicos.ufrn.br/index.php/cronos/article/view/2150/pdf.

MEDRADO, B., \& GALINDO, W. Psicologia social e seus movimentos: 30 anos de ABRAPSO. Recife: Editora Universitária da UFPE,2011.

MORAWSKI, J. Practicing feminisms, reconstructing psychology: notes on a liminal science. Ann Harbour: University of Michigan Press, 1994.

MOSCOVICI, S., \& MARKOVÁ, I. The making of modern social psychology: the hidden story of how an international social science was created. Cambridge: Polity Press, 2006.

PERUCCHI, J. Dos estudos de gênero às teorias queer: desdobramentos do feminismo e do movimento LGBT na psicologia social. Anais do Encontro Nacional da Associação Brasileira de Psicologia Social. Maceió,2009. Recuperado em 2 ago 2013, disponível em: http://abrapso.org.br/siteprincipal/images/Anais_XVENABRAPSO/627.\%20dos\%20estudos $\% 20 \mathrm{de} \% 20 \mathrm{~g} \%$ CAnero $\% 20 \% \mathrm{C} 0 \mathrm{~s} \% 20$ teorias $\% 20$ queer.pdf.

RODRIGUES, A. Sobre o desconhecimento das aplicações da psicologia social. Psicologia: Teoria e Pesquisa, 2(1), 42-55, 1986.

RODRIGUES, A., ASSMAR, E. M. L., \& JABLONSKI, B. Psicologia social. Petrópolis: Vozes, 2003.

SCOTT, J. W. Gênero: uma categoria útil de análise histórica. Educação e Realidade, 20(2), 71-99, 1995.

STREY, M. N. Gênero. In M. G. C. JACQUES, M. N. STREY, N. M. G. BERNARDES, P. A. GUARESCHI, S. A. CARLOS \& T. M. G. FONSECA (Orgs.), Psicologia social contemporânea. Petrópolis: Vozes, 2002.

TONELI, M. J., \& AMARAL, M. S. Gênero, sexo e corpo travesti: abjeções e devires. In B. MEDRADO \& W. GALINDO (Orgs.), Psicologia social e seus movimentos: 30 anos de ABRAPSO. Recife: Editora Universitária da UFPE, 2011.

TORRES, C. V., \& NEIVA, E. R. Psicologia social: principais temas e vertentes. Porto Alegre: ArtMed, 2011.

VALSINER, J. Fundamentos da psicologia cultural: mundos da mente, mundos da vida. Porto Alegre: Artmed,2012.

Data de recebimento: 03/03/14

Data de aceite: $23 / 06 / 15$ 


\section{Sobre as autoras:}

Jaqueline Gomes de Jesus é Pós-Doutora pela Escola Superior de Ciências Sociais da Fundação Getúlio Vargas - CPDOC/FGV, Rio de Janeiro, e Doutora em Psicologia Social e do Trabalho pela Universidade de Brasília - UnB. Vinculação institucional: Psicóloga da Universidade Federal do Rio de Janeiro - UFRJ e Conselheira do Conselho Regional de Psicologia do Distrito Federal - CRP/DF. Endereço eletrônico: jaquebrasilia@gmail.com.

Ana Lúcia Galinkin é pós-doutora em Psicologia Social pela Universidade René Descartes, Paris, França, Doutora em Sociologia pela Universidade de São Paulo, Psicóloga pela UFMG - Vinculação institucional: Professora Associada no Programa de Pós Graduação em Psicologia Social do Trabalho e das Organizações da Universidade de Brasília - UnB. Endereço eletrônico: anagalinkin@gmail.com 